\title{
Consecuencias sociales de un desastre inducido, subsidencia
}

\author{
Ramiro Rodríguez-Castillo*, Isaías Rodríguez-Velázquez \\ Instituto de Geofísica, Universidad Nacional Autónoma de México, México \\ Ciudad Universitaria, Coyoacán, 04510, México, D.F. \\ *rrdz@geofisica.unam.mx
}

\begin{abstract}
Resumen
Los fuertes regimenes de extracción de sistemas acuíferos pueden producir hundimientos diferenciales del terreno. Estos causan fracturas y fallamiento por subsidencia en el terreno dañando la infraestructura urbana y particular. Sien embargo, en México no existen programas oficiales de ayuda a los afectados a ningún nivel dado que la subsidencia no es considerada como desastre. En el presente trabajo se proponen algunas acciones para mitigar los efectos adversos en zonas urbanas y se analiza el problema de la subsidencia y sus repercusiones ambientales, sociales y económicas.
\end{abstract}

Palabras clave: subsidencia, sobreexplotación acuífera, Irapuato, Guanajuato.

\section{Abstract}

The intense extraction regimes of aquifer systems can induce subsidence. Such differential movements cause fractures and faults in terrains affecting urban and private infrastructure. However, in Mexico there are no official paliative programs because subsidence is not considered a disaster. In this paper, subsidence and its environmental, social and economics consequences are analyzed and some mitigation actions for damage due to subsidence in urban areas are proposed.

Key words: subsidence, aquifer overexploitation, Irapuato, Guanajuato.

\section{Introducción}

El abastecimiento nacional de agua para uso urbano, agrícola e industrial se produce en buena medida a partir de agua subterránea. Se estima que del 60 al $65 \%$ del total de agua consumida en el país proviene de sistemas acuíferos, muchos de ellos en estado de sobreexplotación. El mayor consumidor de agua en México es la agricultura, con cerca del $80 \%$ del total.

Los programas y proyectos de abastecimiento no son por lo general planificados en función de la capacidad de respuesta del medio geológico. Una extracción mayor que la recarga, parámetro difícil de calcular, origina abatimientos del nivel piezométrico y merman los volúmenes disponibles. La sobreexplotación obliga a buscar fuentes alternas y a considerar esquemas para un mejor manejo de los recursos, aunque hay otros efectos colaterales de la "inadecuada" explotación acuífera. Uno de ellos es la subsidencia, que se expresa como hundimientos diferenciales del terreno. Esta se asocia comúnmente a sobreexplotación de acuíferos, aunque no necesariamente, ya que tiene que ver mas con la presencia de acuitardos que con la sobreexplotación. Las velocidades de los hundimientos son de varios milímetros a varios centímetros por año.

La alta dependencia en el abastecimiento de agua subterránea provoca que la extracción induzca flujos de acuitardos, despresurizándolos y generando la compactación de los terrenos, proceso que va acompañado de la aparición en la superficie de fracturas algunas de las cuales, por su extensión son llamadas localmente fallas (Figura 1). 
En ciudades como Irapuato, en el Estado de Guanajuato, se tienen identificados hasta 15 sistemas de fallas, con una extensión lineal total de mas de $25 \mathrm{~km}$. (Rodríguez et al., 2006) (Figura 2). En Salamanca, Gto., se han medido velocidades de subsidencia de $6 \mathrm{~cm}$. por año (Garduño et al., 2000).

\section{Causas de la subsidencia}

Los primeros reportes que relacionan los hundimientos del terreno con la extracción de agua fueron realizados por R. Fuller en 1908 (Poland, 1984). El primero en tratar de explicar científicamente el fenómeno fue Terzaghi (1925) quien propone una ecuación de consolidación unidimensional. Dicho trabajo fue retomado por Meinzer (1928) quien reconoció que la extracción de agua del almacenamiento de un acuífero se debe a la compresión del mismo y que la disminución del almacenamiento puede ser permanente (inelástica) o recuperable (elástica). Las primeras observaciones son realizadas por Rappley y Tibbets en 1933 (citados en Poland e Ireland, 1988) en el Valle de Santa Clara en California. Posteriormente, T. Althouse asoció la extracción de agua con hundimientos en el valle de

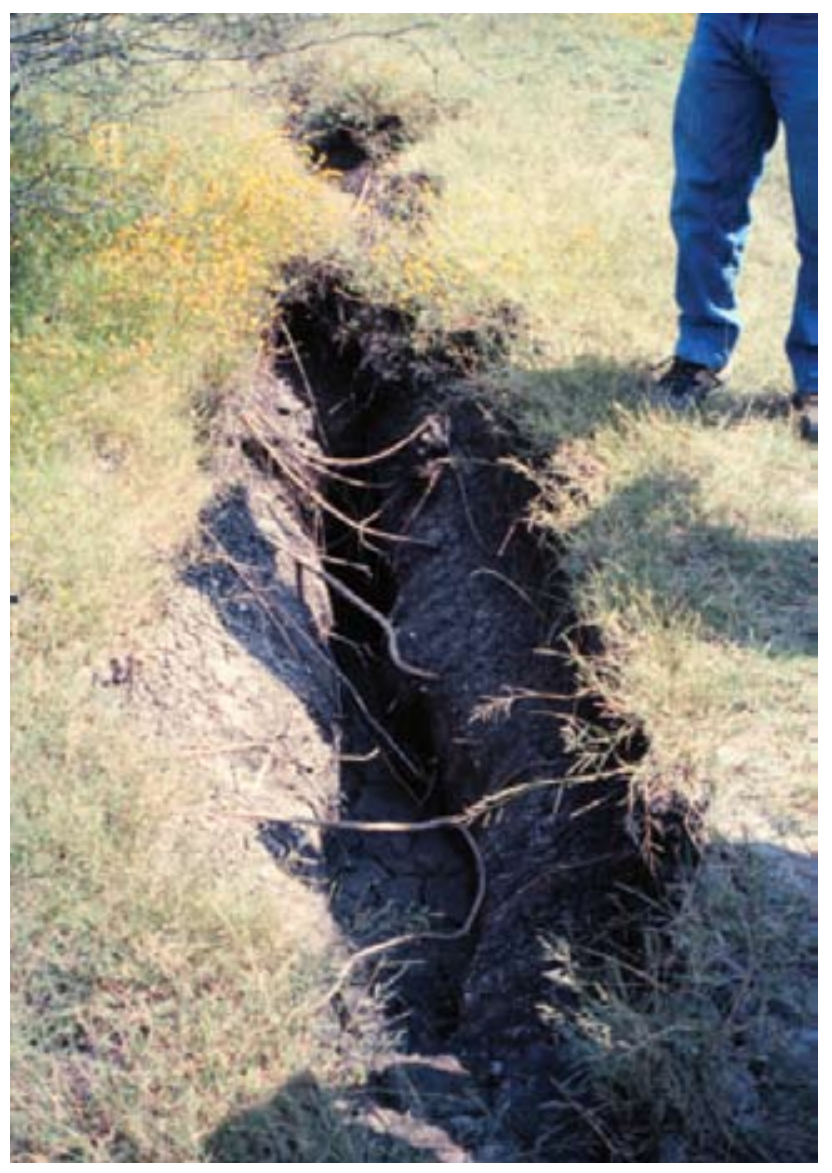

Figura 1. Falla por subsidencia en Salamanca Guanajuato, México.
San Joaquín en California en 1935 (en Evans, 1986). En México, Nabor Carrillo desde la década de 1950 reconoció que la subsidencia esta asociada a la extracción de agua subterránea de el gran paquete arcilloso de origen lacustre de la Cuenca de México (Marsal y Mazari, 1959).

En ciudades afectadas por subsidencia es difícil discernir en qué grado la componente de la extracción de agua subterránea está induciendo los hundimientos. En el Altiplano Mexicano el número de pozos agrícolas supera hasta en dos órdenes de magnitud a los urbanos, lo que se refleja en los volúmenes extraídos. Esta extracción de agua subterránea para uso agrícola podría contribuir en la subsidencia urbana.

\section{3. ¿Se debe considerar a la subsidencia como un desastre?}

Como la subsidencia no esta considerada como un desastre natural ni inducido, ni en la Ley de Aguas Nacionales ni en la Ley General del Equilibrio Ecológico y la Protección al Ambiente (LGEEPA), no se incluye en los programas urgentes de ayuda social. Una razón de tal exclusión es que los efectos por lo general se observan a largo plazo. De esta forma, los afectados por fenómenos de subsidencia no cuentan con elementos legales para ampararse o reclamar.

La subsidencia no sólo se afecta la infraestructura visible sino también a tuberías, drenajes y poliductos. La ruptura de tuberías de agua potable incorpora rápidamente cloro residual a través del fracturamiento del terreno a formaciones acuíferas someras. Desde los drenajes rotos migran fácilmente microorganismos y materia orgánica. Además, cuando las fallas interceptan poliductos se pueden producir derrames de hidrocarburos en el subsuelo.

Los edificios de uso público con estructuras debilitadas por las fracturas deben ser considerados en riesgo ya que son mas fácilmente afectables por sismos o inundaciones. Igualmente edificios y monumentos históricos se ven dañados, como es el caso del Ex-Convento de San Agustín en Salamanca y del Puente Guadalupe en Irapuato, Gto.

Las fallas pueden facilitar también la migración de gas radón hacia la superficie. Aunque su vida media es relativamente corta, del orden de días, la exposición por periodos prolongados al mismo puede afectar seriamente la salud.

\section{Impacto social de la subsidencia}

Los daños a edificios y casas habitación son asimilados por los afectados, llegándose a "pérdidas totales" en la construcción. Se rompen paredes y pisos y se colapsan los techos. Los esfuerzos a que son sometidas las estructuras rompen trabes y cimentaciones (Figura 3). Los afectados realizan contínuas y costosas reparaciones, sin saber que el proceso no se detendrá. Asimismo el precio de los terrenos 


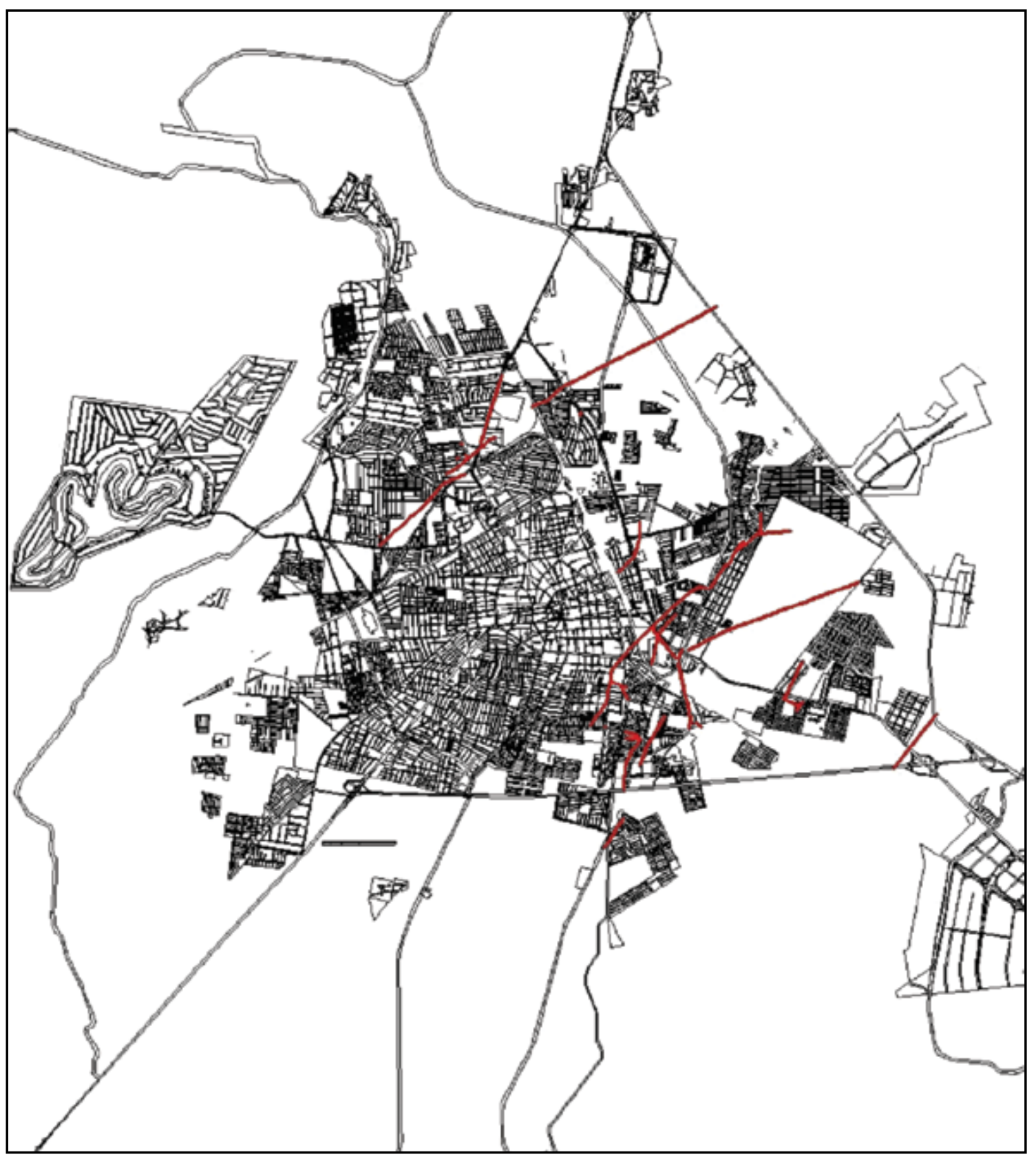

Figura 2. Fallas por subsidencia, en rojo, localizadas en el núcleo urbano de Irapuato, Guanajuato, México.

baja de manera considerable. Naturalmente, la pérdida del patrimonio afecta la calidad de vida de los afectados y crea un problema social.

Igualmente, los costos que deben de asumir los minicipios y estados ante los efectos de la subsidencia son considerables. Tal es el caso, por ejemplo, de los costos de la renivelación de las líneas del Metro y del Drenaje Profundo en la Ciudad de México. El desbordamiento del Río de los Remedios y la inundación de las zonas habitacionales vecinas en Chalco también se deben a la subsidencia, ya que el nivel del cauce del río quedo varios metros por arriba del nivel del terreno.
Si las edificaciones pertenecen a grupos inmobiliarios, éstos difícilmente se hacen responsables. Su excusa es simple: los permisos de construcción los otorgó el Municipio. Sin embargo los encargados de aprobarlos se apoyan en lo que normativamente se exige, que son estudios de mecánica de suelos. Sin embargo, si la subsidencia no es evidente los estudios de mecánica de suelos requeridos para los permisos no la detectarán. Por otro lado, si no existen reglamentos de construcción locales en los cuales se especifiquen acciones concretas en caso de la presencia de fallas o fracturas en el terreno, las reclamaciones no proceden. El agua es un bien nacional de alcance federal, cuya competencia está en la 


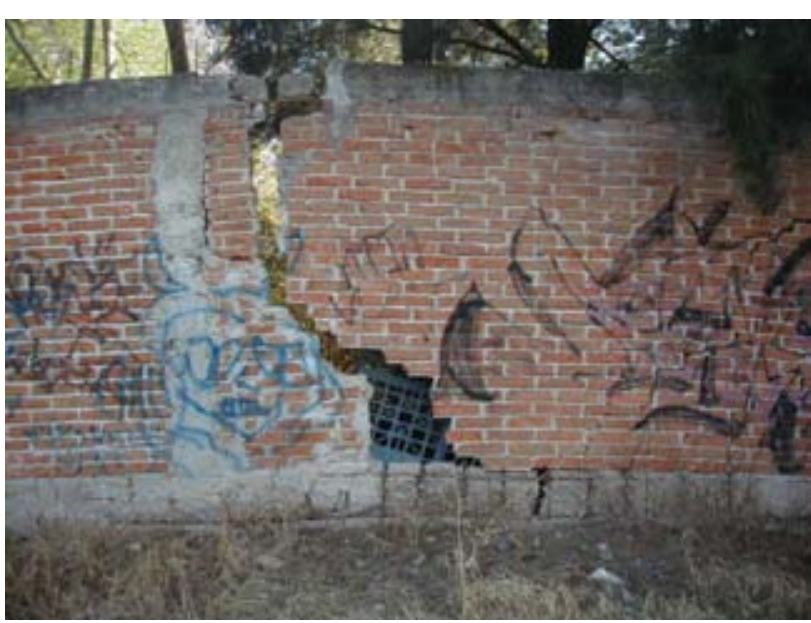

Figura 3. Afectaciones estructurales por subsidencia en Irapuato, Guanajuato, México.

Comisión Nacional del Agua o en las Comisiones Estatales, pero aun ante estas instancias no hay una forma clara de proceder cuando hay problemas debidos a subsidencia. Los grupos de Protección Civil tampoco tienen claro si la subsidencia es un desastre o un fenómeno natural. Con todo ello, no resta ningún orgnismo público ni privado que responda adecuadamente a los afectados.

Existen vacíos legales que deben ser cubiertos para minimizar el impacto económico que tiene la subsidencia. En los llamados Atlas de Riesgos que coordinan encargados de Protección Civil se llegan a incluir mapas con fallas y fracturas del terreno, pero esta información no permea hacia las instituciones encargadas de la obra civil.

Aunque la subsidencia sea inducida, los propietarios de pozos que la provocan no conocen los efectos que la extracción del agua que se les concesionó produce. Los Municipios que reciben reclamaciones pueden argumentar que el agua es un bien federal y que ellos sólo lo administran. Los quejosos son entonces direccionados hacia el ámbito federal, pero nada ampara el reclamo. ¿Los agricultores que usan agua subterránea son los responsables de los efectos dañinos de dicha extracción o lo son las autoridades que asignaron concesiones y volúmenes?

\section{Acciones preventivas. ¿Quién financia?}

Si se entiende el proceso que origina la subsidencia se puede tratar de controlarlo mediante una extracción de agua planificada. Ello requiere de la incorporación de herramientas computacionales como modelos matemáticos de flujo que consideren predicciones de compactación del terreno. Los Municipios deben incorporar la cultura de la prevención y el conocimiento público de fenómenos potencialmente riesgosos para la propiedad inmueble pública o privada. Sistemas de monitoreo de hundimientos deberían de ser práctica común.
En algunos casos, el cambio de ubicación de pozos evita la extracción de los acuitardos o bien se profundizan para minimizar el impacto de la extracción. La alta dependencia de agua subterránea para abastecimiento urbano y para riego agrícola hace prácticamente imposible el suspender la extracción. Los Municipios deben incorporar la subsidencia en los programas de ordenamientos territoriales, limitando drásticamente la construcción en zonas de fallamiento o fracturamiento.

Al estar asociada a la extracción de agua subterránea, la subsidencia es un fenómeno que no se puede detener mientras continue la explotación de los sistemas acuíferos regionales. La recarga artificial no es una solución ya que las unidades arcillosas no se pueden recargar, aunque puede ser un recurso paliativo en aquellos casos en donde los acuitardos tengan componentes subyacentes factibles de ser recargados.

Para manejar de la manera más adecuada el problema son necesarias las siguientes acciones:

- Mapeo de las fallas o sistemas de fracturas

- Geoposicionamiento de las fallas o fracturas en planos urbanos escalas 1:10,000 0 1:25,000, verificando con estaciones totales su posición.

- Creación de bases de datos geológicos y de información sobre número de pozos y volúmenes de extracción

- Creación de Sistemas de Información Geográfica que permitan la rápida incorporación de información nueva

- Establecimiento de un programa de monitoreo para observar la evolución espacial y temporal de los desplazamientos verticales.

- Definición de perímetros de riesgo

- Establecimiento de contacto permanente de los grupos que estudien la subsidencia con las dependencias locales encargadas de permisos de construcción.

Una primera acción correctiva es la separación estructural de las edificaciones dañadas de las que no lo están para evitar que estas obras se afecten. Las redes de tuberías tanto de agua potable como de drenaje deben de rediseñarse evitando las calles en donde crucen fallas o substituirse por ductos de material no rígido, que tolere la deformación.

\section{Conclusiones}

Aunque existan evidencias de ello, no es fácil demostrar que un proceso de hundimiento del terreno, o subsidencia, esté estrechamente relacionado con la extracción de agua subterránea, extracción que mayoritariamente no es urbana. Aun en el caso de que la extracción sea mayoritariamente agrícola, no se puede "culpar" a la misma de los hundimientos pues los usuarios agrícolas poseen concesiones asignadas por autoridades federales.

Dada la desprotección oficial de los afectados por la 
subsidencia, la única alternativa que tiene la sociedad es protestar a través de los medios de comunicación masivos, organizaciones ambientalistas o partidos políticos que no constituyen instancias legales adecuadas para atacar el problema ni para paliar sus efectos. Por ello, es preciso que las instituciones federales delimiten responsabilidades sobre los problemas derivados de la subsidencia.

Es necesario legislar al respecto e incorporar información técnica como mapas de riesgos, de vulnerabilidad acuífera o Sistemas de Información Geográfica en los organismos dedicados al agua, al medio ambiente, a la protección civil o la obra e infraestructura urbana. La incorporación de herramientas como modelos matemáticos computacionales y levantamientos topográficos periódicos con estaciones totales son imperativos zonas en donde se ha reportado subsidencia.

\section{Agradecimientos}

Esta investigación se desarrolló con apoyo financiero del Proyecto Concyteg GTO-04-C02-113 y de la JAPAMI Irapuato, Gto.

\section{Referencias bibliográficas}

Evans, R. S., 1986. A Regional Groundwater model for Open Cut Coal Winning in the Latrobe Valley, Victoria. AWRC Conference, Groundwater Systems Under Stress, Brisbane.

Garduño V. H., Arreygue E y Rodríguez G., 2000. Mapa de riesgos de Salamanca. Reporte Técnico. Municipio de Salamanca, Univ. Nicolaita Michoacán.

Marsal R. J. y Mazari M., 1959. El subsuelo de la Cd. de México: México D. F. UNAM, Fac. de Ingenieria, Vol I y II, 505 pp.

Meinzer O. E., 1928. Compressibility and elasticity of artesian aquifers. Econ. Geol. Vol. 23, Num 3. 263-291 pp.

Poland, J. F., 1984. Guidebook to Studies of Land Subsidence Due to Groundwater Withdrawal. UNESCO Studies and Reports in Hydrology no. 40. New York; Unipublications.

Poland, J. F., and R. L. Ireland, 1988. Land Subsidence in the Santa Clara valley, California, as of 1982, Mechanics of Aquifer Systems, U. S. Geol. Surv. Prof. Pap., 497-F,61 pp.

Rodriguez R., Armienta A., Morales P, Silva T y Hernández H., 2006; Evaluación de Vulnerabilidad Acuífera del valle de Irapuato Gto. JAPAMI, CONCyTEG, IGF UNAM. Reporte Técnico il. 90 pp.

Terzaghi, K., 1925, Principals of Soil Mechanics 1V: Settlement and consolidation of clay: Engineering News-Record, v. 95 (Nov 26), p. $874-878$.

Manuscrito recibido: Enero 17, 2007

Manuscrito corregido recibido: Enero 25, 2007

Manuscrito aceptado: Enero 31, 2007 